\title{
L'acharnement apologétique : Arendt au secours de Heidegger
}

Note critique sur Emmanuel FAYE (2016). Arendt et Heidegger.

Extermination nazie et déstruction de la pensée. Paris, Albin Michel

The Relentless Praise : Arendt to the Rescue of Heidegger. Review of Emmanuel Faye (2016). Arendt et Heidegger. Extermination nazie et destruction de la pensée. Paris, Albin Michel

El enseñamiento apologético : Arendt al rescate de Heidegger. Nota crítica sobre Emmanuel Faye (2016). Arendt et Heidegger. Extermination nazie et destruction de la pensée. Paris, Albin Michel

\section{Michel Baudouin}

\section{(2) OpenEdition}

\section{Journals}

Édition électronique

URL : http://journals.openedition.org/bssg/151

DOI : $10.4000 /$ bssg. 151

ISSN : 2490-9424

Éditeur

Presses universitaires de Vincennes

Référence électronique

Michel Baudouin, «L'acharnement apologétique : Arendt au secours de Heidegger », Biens Symboliques / Symbolic Goods [En ligne], 1 | 2017, mis en ligne le 15 octobre 2017, consulté le 04 mars 2021. URL: http://journals.openedition.org/bssg/151 ; DOl : https://doi.org/10.4000/bssg.151 

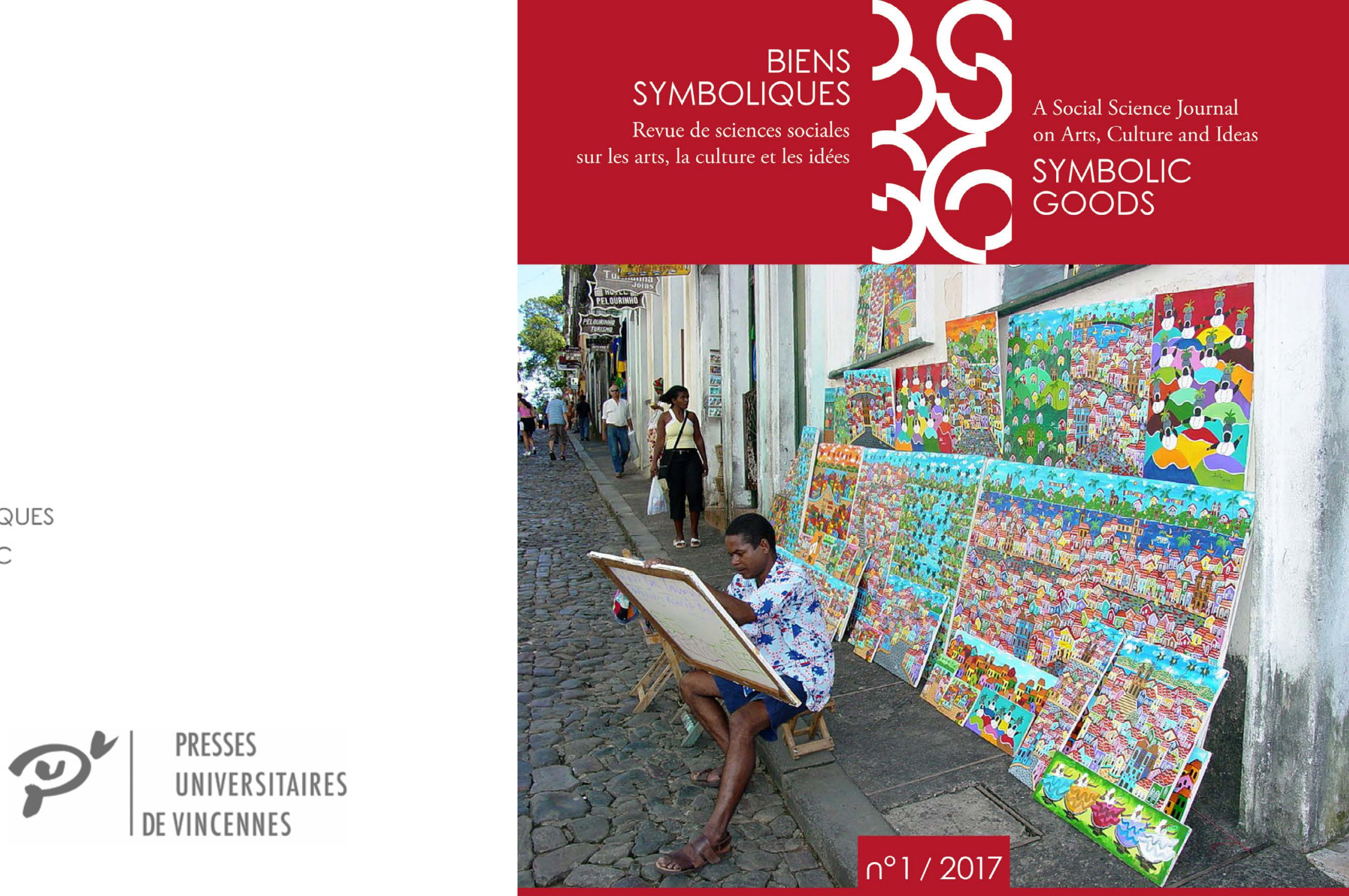

\section{Artistes ordinaires \\ Ordinary Artists}




\section{L'acharnement apologétique : Arendt au secours de Heidegger \\ Note critique sur Emmanuel Faye (2016). Arendt et Heidegger. Extermination nazie et déstruction de la pensée. Paris, Albin Michel}

Michel Baudoin

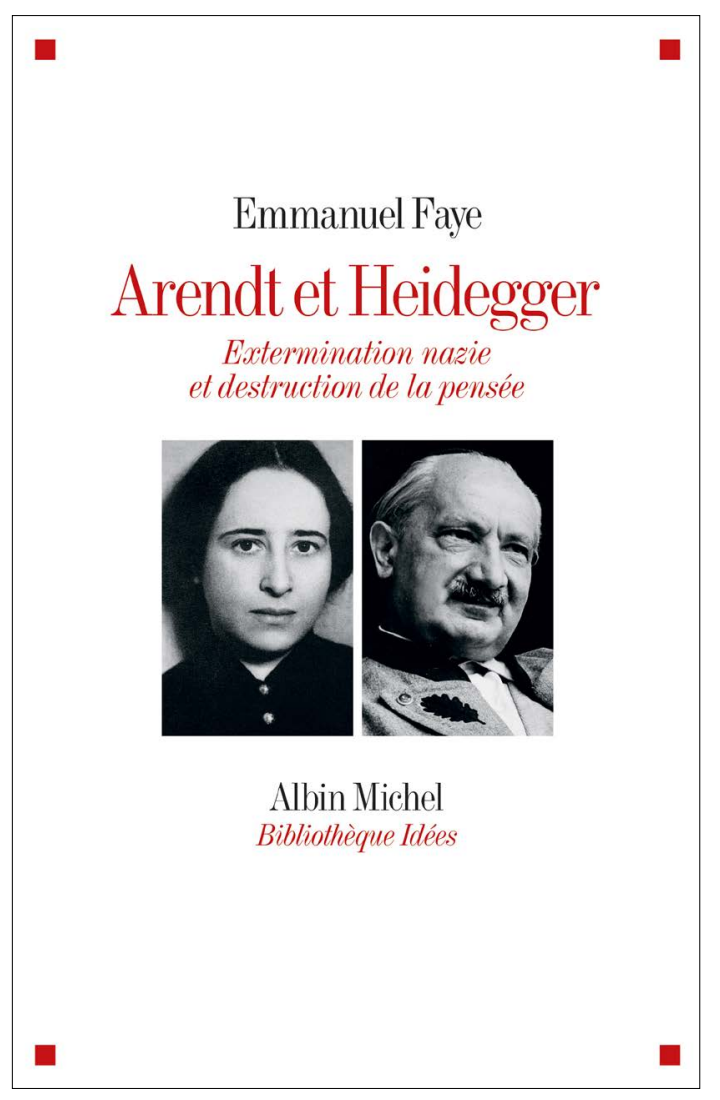

(c) Paris, Albin Michel [http://www.albin-michel.fr/ouvrages/arendt-et-heidegger-9782226315137].
Depuis les années 1930, la célébrité de Heidegger semble autant reposer sur l'influence énorme exercée par sa pensée que sur son engagement au côté des nazis. Si cet engagement n'a pas disqualifié a priori l'importance accordée à cette pensée, c'est en partie du fait que sa durée et son intensité ont été longtemps minimisées par Heidegger lui-même et ses disciples : le «Maître ${ }^{1}$ » n'aurait cédé aux sirènes de la révolution conservatrice que durant les quelques mois où, en 1933, il a accepté d'être recteur de l'université de Fribourg avant de démissionner du fait d'un désaccord profond avec les nazis ${ }^{2}$. Dans ces conditions, il était raisonnable de n'y voir qu'une erreur liée à des circonstances difficiles sans conséquence pour la pensée heideggerienne.

Certains nourrissaient cependant déjà davantage que quelques soupçons, allant jusqu'à affirmer un lien fort entre la pensée du philosophe et son engagement politique. Le philosophe allemand Karl Loewith, ancien étudiant et proche de Heidegger (Janicaud 2001 : 120-134), le sociologue

Ainsi que le désigne malicieusement François Rastier (2015).

2 Heidegger, Entretien donné au Spiegel en 1966, publié en 1976. C'est encore plus ou moins la version des faits proposée par Dominique Janicaud pourtant heideggerien modéré, qui parle de « l'engagement de 1933 » dans son ouvrage Heidegger en France (Janicaud 2001). 
Georges Gurvitch (Faye 2016 : 276-277, note 3), et le philosophe Alexandre Koyré (Faye 2016 : 324-325) comptent parmi les premiers critiques, dès 1946. Adorno essaie, une vingtaine d'années plus tard, de montrer que la philosophie de Heidegger se réduit à un envoûtement verbal abusant de l'ontologie pour masquer son idéologie politique (Adorno 2009) ${ }^{3}$. En 1975, dans le cinquième numéro des Actes de la recherche en sciences sociales, Bourdieu soutient à son tour que l'ontologie heideggerienne n'est que le recodage universitaire sublimé et acceptable d'énoncés nazis vulgaires. Mais toutes ces interventions restent relativement souterraines. Le premier grand débat public n'éclate en France qu'en 1987 avec la publication du livre de Victor Farias, Heidegger et le nazisme, où l'on apprend que le "Maître » a participé bien plus activement et longuement à la révolution conservatrice qu'on ne l'imaginait et qu'il ne l'avait avoué. Bien des philosophes heideggeriens sont sans doute ébranlés profondément par ces révélations. Mais la pensée heideggerienne elle-même leur semble encore trop profonde et riche pour être brutalement réduite au nazisme de son auteur ${ }^{4}$

C'est pourquoi le premier livre d'Emmanuel Faye sur le sujet, Heidegger, l'introduction du nazisme dans la philosophie, fait

3 La traduction tardive, en 1989, de cette œuvre parue en 1964 (par une heideggerienne, Éliane Escoubas, qui démine son contenu en essayant de rapprocher Adorno et Heidegger dans la préface) est regrettable. Est-ce la raison pour laquelle Janicaud (2001), bien qu'il la mentionne, ne la discute pas?

4 Janicaud (2001: 348-390) fait un compte rendu de la querelle relativement équilibré, même s'il finit par pencher en faveur de Heidegger et que les principaux points de sa défense sont aujourd'hui affaiblis, si ce n'est anéantis, par les travaux réalisés par Emmanuel Faye depuis 2005. grandbruiten2005. EmmanuelFaye ydémontre, mais cettefois grâce à des cours inédits dont l'accès n'était pas contrôlé par les exécuteurs testamentaires du « Maître », que la philosophie de Heidegger est à ce point imprégnée d'antisémitisme et d'hitlérisme qu'il s'agit moins d'une philosophie que d'une œuvre de propagande nazie parée des atours d'une pensée essentielle (Faye 2007 : 25). Confrontées à ces accusations, les autorités philosophiques françaises, armées seulement de leur courage, de leurs œillères et de leurs pince-nez, inscrivent Heidegger au programme de l'agrégation. On n'en attendait pas moins. Préserver l'honneur de la philosophie et la valeur d'échange d'un capital symbolique durement acquis par la lecture laborieuse de textes obscurs et boursouflés nécessitait de caricaturer Emmanuel Faye en FouquierTinville de la philosophie, de l'accuser à tort de vouloir détruire la philosophie et les livres de Heidegger. La publication, en 2014, des Cahiers noirs, prévue par Heidegger lui-même comme le couronnement de ses œuvres complètes, assène enfin aux indéfectibles défenseurs de Heidegger un coup violent ${ }^{5}$. Jusqu'ici les heideggeriens pouvaient encore insister sur l'absence d'antisémitisme dans les écrits philosophiques du « Maître » - en omettant toutes les références implicites, contenues dans les œuvres publiées, à la figure du Juif sous les guises du changeur, du déraciné ou de l'Asiatique ainsi que les références à peine cryptées à des antisémites aussi notoires à cette époque que le comte de York. Les

5 Ainsi Stéphane Domeracki, jusqu'alors heideggerien, a tourné casaque. On peut consulter avec profit son ouvrage, Heidegger et sa solution finale (Domeracki 2016), qui contient de nombreuses traductions des Cahiers noirs, qui n'ont toujours pas été traduits en français. 
Cahiers noirs détruisent ce dernier bastion en explicitant un antisémitisme pensé et assumé. Heidegger y écrit, entre autres choses que "les Juifs, avec leur talent marqué pour le calcul, "vivent" depuis le plus longtemps déjà d'après le principe de la race, ce qui explique pourquoi ils résistent à sa pleine application avec la plus grande violence ${ }^{6} »$. Autrement dit, les Juifs seraient les responsables du traitement qui leur est infligé par les nazis et ils ont de surcroît l'impolitesse d'y résister violemment. Cette ignominie heideggerienne illustre à la perfection ce que Karl Kraus, le journaliste et polémiste autrichien, écrivait dès 1933 des nazis qui, sans aucune honte ni hésitation, se présentent sous les traits de « cette fameuse innocence qui persécute », où se dévoile «l'unité de la faute et du mensonge où le crime devient alibi et l'horreur motif d'honneur » (Kraus 2005 : 191). Il semble ainsi de plus en plus clair que Heidegger comptait, non sans raison au vu du climat idéologique actuel, sur un affaiblissement du souvenir des horreurs nazies pour pouvoir, de façon posthume, repasser d'un régime discursif d'insinuation à un régime d'affirmation explicite, comme il avait pu le faire de son vivant avec l'arrivée au pouvoir de cet homme dont il a rêvé de se rapprocher et dont il trouvait qu'il avait d'« admirables mains » (Faye 2007 : 340), Hitler.

Emmanuel Faye considère donc désormais que l'affaire Heidegger est entendue, que les études critiques sont bien établies et qu'il est temps d'examiner la réception de la pensée heideggerienne, les raisons de son ampleur et ses conséquences. L'ouvrage de Janicaud n'avait abordé la réception de Heidegger que dans un cadre français. Or

6 GA $96: 56$, traduit et cité in Domeracki $2016: 25$.
Heidegger est étudié dans le monde entier et c'est sans doute en partie grâce à Hannah Arendt qui est, selon Emmanuel Faye, « la figure qui aura le plus contribué après 1945, à la diffusion planétaire de la pensée de Heidegger » (Faye 2016 : 12). Sans elle, il n'aurait sans doute pas pu construire sa réputation de plus grand penseur $\mathrm{du} x \mathrm{x}^{\mathrm{e}}$ siècle. Mais alors un problème saute aux yeux : « comment le même auteur a-t-il pu concilier la défense hyperbolique de Heidegger et la description critique du totalitarisme national-socialiste ? " (Faye 2016 : 12-13) Arendt ayant été non seulement l'étudiante mais aussi l'amante de Heidegger au milieu des années 1920, beaucoup ont été tentés par l'explication romantique et sexiste : l'amour aurait partiellement aveuglé Arendt face aux errements politiques du "Maître », bien qu'elle ait opérée une critique subtile des thèses de Heidegger dans son œuvre. Faye soutient une interprétation tout autre. Si Arendt s'éloigne de l'univers intellectuel de Heidegger durant les années 1930 et l'essentiel des années 1940, elle devient pleinement heideggerienne entre 1947 et 1949 (donc avant même de revoir le "Maître » en Allemagne en février 1950) sans doute à cause de l'attrait philosophique exercé sur elle par la Lettre sur l'humanisme, écrite par Heidegger en réponse à des questions posées par Jean Beaufret ${ }^{7}$ et dont elle admire la violence à l'égard de la tradition occidentale. À partir de là, Arendt va s'employer à innocenter les élites intellectuelles allemandes nazies des crimes commis par leur

7 Jean Beaufret (1907-1982), résistant, professeur de philosophie, principal interlocuteur de Heidegger en France après 1945, formateur de nombreux heideggeriens influents, tels que François Fédier (exécuteur testamentaire et traducteur de Heidegger), mais aussi de Robert Faurisson auquel il a apporté son soutien à la fin de sa vie. 
parti en proposant un schéma d'explications encore répandu aujourd'hui malgré les lacunes et les ambiguïtés liées au fait qu'elle s'inspire souvent d'auteurs directement impliqués dans le mouvement nazi (Heidegger donc, mais aussi Carl Schmitt, Walter Franck, Hermann Rauschning, etc.).

Ces ambiguïtés sont surprenantes. Arendt est une victime du régime nazi. II s'en est fallu de peu qu'elle ne lui survive pas. Mais si elle s'est opposée de manière déterminée à l'antisémitisme qui sévissait en Allemagne, elle a aussi manifesté assez tôt un intérêt pour des thèses contrerévolutionnaires et conservatrices. Au début des années 1930, avant d'être obligée à l'exil par la Gestapo, Arendt étudie Adam Müller (1779-1829), un critique littéraire romantique, penseur de l'État et admirateur de Burke, en passe d'être récupéré par les nazis (Faye 2016 : 61). Si elle tente de montrer que les nazis ne sont pas en droit de se réclamer de Müller, deux idées présentes chez cet auteur la séduisent. D'une part, c'est l'idée classique des anti-Lumières selon laquelle le concept universel de l'Homme doit être récusé du fait de son abstraction qui ne rend pas compte de la diversité concrète des hommes (l'idée même de droits humains universels est absurde de ce point de vue et c'est bien là le but de cette récusation). D'autre part, Arendt retient aussi l'importance du concept tout à la fois biologique, historique et religieux, de « communauté ». Selon Müller, ce n'est qu'à travers la communauté que l'individu peut trouver son salut. D'après Emmanuel Faye, Arendt souscrit à ces idées jusque dans ses dernières œuvres et elle ne défend ni la valeur individuelle ni la dimension universelle de l'être humain (Faye 2016 : 63). À la fin des années 1930, Arendt, alors en exil à Paris, n'est toutefois pas encore encline à innocenter les intellectuels allemands. Dans un essai inachevé, Antisemitismus, Arendt dénonce dans l'antisémitisme des intellectuels romantiques allemands l'origine de l'antisémitisme moderne (Faye 2016 : 68-71).

Arendt suggère même que Heidegger aurait sa place dans cette généalogie, lui qu'elle désignera comme le dernier des romantiques en 1946, dans son article intitulé Qu'estce que la philosophie de l'existence ? À cette date, Arendt reproche encore à Heidegger de détruire l'humanisme dans la mesure où il opposerait le « soi » et le « on » sans donner aucun contenu humain particulier au « soi » (285-303). Or dès 1927, dans Être et temps (§ 74), Heidegger montre que le soi authentique ne s'accomplit que dans la communauté du peuple, uni dans un destin commun né de l'anticipation de la mort et scellé par la poursuite du combat et le choix de son héros. Arendt sait tout cela, mais elle fait comme si ces thèmes suspects étaient apparus après Être et temps. Et si elle mentionne l'engagement nazi de Heidegger en passant, elle le tourne tellement en dérision que son ancien maître est disculpé par son propre ridicule. Mais bientôt elle sera elle aussi séduite par la destruction de l'humanisme.

Plus tard dans l'année 1946, la recension critique de deux ouvrages, un collectif, Le Livre noir : le crime nazi contre le peuple juif, et un de Max Weinreich, Les Professeurs de Hitler, infléchit en effet significativement la position d'Arendt (Faye 2016 : 19-48). Le Livre Noir, contient un livre à part entière, L'Enfer de Treblinka, écrit par Vassili Grossman, qui décrit l'irréductible humanité des déportés manifestée dans la résistance armée et la solidarité face à l'extermination. Or Arendt ne le mentionne pas et emploie des arguments aussi ambigus que promis à un avenir certain. Dans sa présentation, 
les déportés sont déshumanisés, égaux seulement comme les animaux d'un troupeau passif destiné à ces usines de production des cadavres que sont les camps d'extermination, comme s'il était justifiable de leur reprocher de s'être laissé faire (ce qui est faux et/ou ignoble), comme si tuer des êtres humains avant de faire disparaître leurs corps était comparable à la production d'un objet. Heidegger lui-même utilisera cette image industrielle pour diluer la singulière horreur des camps et en attribuer la responsabilité à la civilisation scientifique et rationaliste que les nazis eux-mêmes haïssaient. Arendt va jusqu'à présenter l'extermination comme un crime si terrible qu'il ne laisse derrière lui ni bourreaux ni victimes - puisque, dans ce tourbillon meurtrier, Arendt prétend que les rôles sont interchangeables.

D'autre part, si Arendt commence par reconnaître quelques qualités au livre de Weinreich qui défend la thèse que les savants allemands ont fourni les idées, les techniques et les justifications du génocide, c'est pour finir sur une critique sans appel. Le fait qu'Heidegger soit mentionné par Weinreich et associé à Eugen Fischer, le directeur de l'institut de Berlin où sera formé Joseph Mengele, médecin d'Auschwitz, n'y est pas, selon Emmanuel Faye, étranger (Faye $2016: 35)^{8}$. Arendt accorde encore (ce qu'elle ne fera plus cinq ans plus tard) que Schmitt, Heidegger et quelques autres sont allés au-delà de la mesure dans leur soutien au régime hitlérien, mais elle ne les qualifie pas eux-mêmes de nazis. Cette concession faite, elle affirme que les nazis ne s'intéressaient pas aux vrais penseurs, mais seulement aux techniciens sans

8 Sur la très longue amitié entre Heidegger et Fischer, voir Faye 2007 : 77, mais aussi Janicaud $2001: 354$. idées. Les intellectuels nazis sont donc totalement disculpés, la populace et les politiciens accusés. Cette conception sera développée plus tard par Arendt et elle servira de caution à tous ceux qui soutiennent que le nazisme n'a été défendu que par des intellectuels de seconde zone et non pas par de grands penseurs.

C'est en 1949 qu'Arendt achève de se convertir aux idées heideggeriennes. Emmanuel Faye en trouve la preuve dans la correspondance d'Arendt avec Dolf Sternberger, un philosophe proche de l'école de Francfort que le régime nazi avait contraint à abandonner la carrière universitaire au profit du journalisme parce qu'il était marié à une juive (Faye 2016 : 308-326). C'est à ce moment que Sternberger demande à Arendt si elle accepterait de rédiger un essai critique consacré à la Lettre sur l'humanisme. II a lu son article de 1946 sur la philosophie de l'existence et il pense qu'elle est la femme de la situation. Arendt répond sèchement qu'elle n'a aucune critique philosophique à opposer à ce texte de Heidegger qu'elle apprécie. Emmanuel Faye en conclut que c'est bien la lecture de Heidegger, et non un sentiment amoureux, qui motive le tournant dans le jugement qu'Arendt porte sur lui. Cela est en partie confirmé par le fait qu'à la même époque Arendt écrit à Jaspers qu'elle est encore furieuse contre Heidegger du fait de son comportement envers Husserl. La publication et le succès des Origines du totalitarisme en 1951 ouvrent à Arendt la porte à toutes les universités nordaméricaines dans le contexte de la guerre froide. Or, dans cet ouvrage, au-delà de quelques différences terminologiques, Arendt ne fait que transposer dans une théorie explicitement politique les concepts et les thèses de Heidegger (Faye 2016 : 346). Jusqu'ici, la plupart des commentateurs d'Arendt 
aveuglés par les petites différences, n'ont pas vu les grosses ressemblances entre elle et le «Maître ». Pourtant, en 1954, elle revendique l'introduction des existentiaux heideggeriens dans la théorie politique. Heidegger aurait ouvert la voie à un réexamen de l'entière sphère politique à la lumière des expériences humaines élémentaires (et qui, fondées dans l'affect, permettent de récuser une approche rationaliste de la politique). Ces expériences humaines élémentaires renvoient justement aux existentiaux déployés un temps par Heidegger dans la description phénoménologique de la quotidienneté. Arendt présente, par exemple, comme Heidegger lui-même, l'expérience fondamentale du totalitarisme sous les espèces de l'abandon et de l'isolement. C'est bien sûr un contresens massif sur le nazisme qui désirait au contraire plus que tout l'unité organique du corps politique. Dans un revirement total par rapport à l'article de 1946 où Heidegger était présenté comme un nihiliste, c'est lui qui nous permet maintenant de penser la pluralité politique grâce à la notion d'être-ensemble. Dans les mêmes années, c'est la logique (et donc la raison) qui, comme la philosophie est opposée par Heidegger à la pensée, est accusée par Arendt d'avoir formée l'idéologie totalitaire : la logique du totalitarisme serait le produit du totalitarisme de la logique elle-même (Faye 2016 : 344). Nous pouvons remarquer ici que cette idée invraisemblable est encore répétée par d'éminents philosophes comme une évidence. C'est Bernard-Henri Levy pour qui le fascisme est le produit des Lumières, c'est Robert Maggiori9 pour qui «le nazisme aveugle encore la philosophie, sans doute parce

9 Libération, 05/02/2015 qu'il présente devant ses yeux la plus horrible construction qu'a pu réaliser son outil le plus précieux : la Raison ».

II n'est donc pas tout à fait étonnant qu'Arendt renonce à accuser le romantisme allemand d'être une des sources de l'antisémitisme nazi, en reprenant une thèse de Carl Schmitt : le romantisme politique aurait été trop relativiste pour développer autre chose qu'un antisémitisme social, inoffensif, opposé à l'antisémitisme politique ultérieur (Faye 2016 : 74). Les véritables origines de l'antisémitisme politique selon Arendt seraient les Juifs eux-mêmes (Faye 2016 : 81), les Lumières françaises, Disraeli ${ }^{10}$ qui aurait été le premier à concevoir la race comme la clé de l'Histoire et qui aurait inspiré Hitler, l'affaire Dreyfus du fait de l'influence qu'Arendt prête à la populace dans une société décomposée où l'élite mafieuse compose avec la masse des déclassés. En 1967, dans la préface tardive au volume sur l'antisémitisme, Arendt accentuera sa thèse de la responsabilité des Juifs dans la formation de l'antisémitisme : ils auraient les premiers défendu la conception raciale du judaïsme, reprise ensuite par les nazis. L'antisémitisme allemand du XIX ${ }^{e}$ siècle n'aurait produit en réalité que le sionisme. Céder à l'envoûtement d'un paradoxe, cela peut arriver. Accuser les principales victimes d'une idéologie meurtrière d'en être les responsables, alors même que l'on fait partie des victimes et non des bourreaux, ne devrait pas laisser beaucoup de place à la bienveillance interprétative.

Le balancier de la responsabilité des crimes nazis antisémites penchant en apparence une fois de plus vers les victimes

10 Benjamin Disraeli (1804-1881), homme politique et écrivain britannique, né dans une famille juive mais élevé dans la foi anglicane. 
elles-mêmes et la populace, il va de soi qu'Arendt doit excuser les élites intellectuelles nazies. Si la disparition des classes sociales constitue un élément essentiel du totalitarisme selon Arendt, elle est bien obligée d'admettre que l'Allemagne nazie est rigidement hiérarchisée, avec l'affirmation d'une forme d'aristocratie, et que la société de classe n'y a pas disparu. C'est la lutte des classes que les nazis rêvent de dissoudre dans le nationalisme fanatique, pas la hiérarchie. Cependant Arendt prétend que l'alliance entre les élites et la populace n'est que provisoire et caractéristique de la phase de la conquête nazie du pouvoir (Faye 2016 : 128). Dès que le pouvoir politique est acquis, ce sont les « masses de philisitins bien organisés » qui s'occupent de tout, en écartant les élites. C'est pourquoi Arendt peut écrire qu'« il faut être juste envers les membres de l'élite qui, à un moment ou à un autre, se sont laissés séduire par les mouvements totalitaires, et qui, à cause de leurs capacités intellectuelles, sont même accusés quelquefois d'avoir inspiré le totalitarisme : ce que ces désespérés du $\mathrm{XX}^{\mathrm{e}}$ siècle ont fait ou non n'eut absolument aucune influence sur le totalitarisme » (cité par Faye 2016 : 129). C'est qu'à ses yeux, le national-socialisme est un mouvement plébéien composé de déclassés manipulés par une pseudo-élite mafieuse dont l'idéologie n'est qu'un prétexte et un instrument dans la conquête du pouvoir. Nul besoin pour le comprendre de lire les nazis... même si elle emprunte sa vision du mouvement totalitaire à Rauschning, lui-même ancien nazi. Or Emmanuel Faye remarque que les nazis n'ont pas pris le pouvoir qu'avec des armes. Leur victoire est aussi liée à leur omniprésence dans les champs littéraire, philosophique, scientifique et culturel (Faye 2016 : 131). II n'est donc pas si simple d'exonérer les intellectuels comme Heidegger de leur responsabilité dans le succès du nazisme.

La Condition de l'homme moderne est publiée en 1958. Quand Arendt l'envoie à Heidegger, elle lui écrit dans la lettre jointe : " pour ainsi dire, [ce livre] te doit tout à tous égards » (cité par Faye 2016 : 360-361). Cela est avéré par la terminologie employée : de la version allemande à la version anglaise, Arendt traduit Dasein par human condition. C'est donc sans surprise qu'un grand nombre de lieux communs heideggeriens trouvent leur place dans le prologue : critique réductrice de la science, dépréciation de la société égalitaire accusée d'empêcher par l'universalité du salariat l'émergence d'une aristocratie intellectuelle ou politique, condamnation de la technique, dénonciation suspecte de l'aliénation, comprise comme Heidegger, par laquelle l'Homme aurait perdu le sens de la Terre et du Monde pour se concentrer sur l'Univers (de Galilée à la conquête de l'espace) et le Moi (Descartes et son subjectivisme individualiste supposé) (Faye 2016 : 368).

Emmanuel Faye avance la thèse convaincante qu'Arendt défend en réalité une vision politique élitiste, aristocratique et sélective inspirée, parfois implicitement, de Heidegger (Faye 2016 : 379-380). Quand le questionnement heideggerien «Qui sommes-nous ? » est introduit à la place de la question universaliste «Qu'est-ce que l'Homme ? », jugée trop théologique, son origine est camouflée par la référence à saint Augustin alors même qu'Arendt y répond d'une façon toute heideggerienne encore : I'humanité est un statut (pas une nature) qui ne peut être conféré que par l'appartenance à une communauté politique déterminée. Mais si cette condition est nécessaire, elle n'est pas suffisante. L'humanité la plus haute est en effet conquise dans l'action politique. C'est ainsi 
qu'à partir de la distinction étymologiquement discutable entre les mots grecs zoe et bios, par exemple, les travailleurs sont renvoyés à la vie animale (zoe) car ils sont tellement occupés à reproduire leurs conditions matérielles d'existence qu'ils deviennent incapables d'accéder à la véritable existence humaine impliquée dans l'action politique (bios). Même la séparation du politique et du social qui en découle, en fait empruntée à Schmitt (Le Concept de politique), est présentée de façon heideggerienne comme un processus historique de décadence partant de l'époque grecque (la plus haute), passant par l'époque romaine (déjà plus basse), pour enfin s'aggraver avec la modernité. Cette décadence serait marquée par la perte progressive de la distinction entre la vie privée et la vie publique. La vie privée consacrée à l'entretien de la vie biologique pouvait être marquée par la violence, ne serait-ce que du fait des esclaves. Or Arendt justifie l'esclavage et la violence privée pré-politique qu'il suppose car cela permet à certains de se libérer des contraintes de la vie biologique pour accéder à la liberté, à l'action politique. L'animalisation des travailleurs est ainsi entérinée. Seuls ceux qui accèdent à la vie proprement humaine dans l'action politique, fondamentalement conflictuelle, sont égaux. Arendt ne comprend en effet pas le combat politique sur un mode délibératif, mais plutôt sur un mode où la violence physique est présente et où il faut risquer sa vie. On serait en droit de se demander d'ailleurs si cette conception agonistique et élitiste de la politique n'est pas une façon détournée de justifier le nazisme de gens comme Heidegger ou Schmitt : eux auraient le mérite d'avoir pris le risque de l'engagement politique, survolant de si haut la populace obsédée par ses besoins vitaux grâce à leur questionnements essentiels sur la communauté qu'on pourrait leur pardonner de s'être si grandement égarés. Ne faudrait-il pas plutôt voir un signe de petitesse morale et intellectuelle dans la fascination éprouvée par ces « grands » penseurs pour la violence politique et dans la stigmatisation obsessionnelle d'une minorité inoffensive? Quoi qu'il en soit, Arendt achèvera la réhabilitation de Heidegger en utilisant le Eichmann largement fictif qu'elle met en scène dans le livre qu'elle a consacré à son procès, Eichmann à Jérusalem publié en 1963 (Faye 2016 : 450457). II est aujourd'hui reconnu qu'Eichmann n'était pas un fonctionnaire terne uniquement occupé de faire circuler avec efficacité les trains remplis de déportés. C'était un nazi authentique, habité par la volonté farouche d'exterminer les Juifs d'Europe. Contrairement à la formule célèbre d'Arendt reprise mécaniquement affirmant la «banalité du mal », le mal inimaginable dont Eichmann est responsable n'est pas le produit d'une activité standardisée et irréfléchie. Mais décrire le nazi type au moyen d'un Eichmann presque idiot, satisfait d'obéir aux ordres sans considérer d'autres conséquences que son avancement dans la hiérarchie, ne peut que confirmer l'innocence des penseurs. Si le véritable nazi est incapable de penser, alors on peut croire que " le roi secret de la pensée », Heidegger, ne peut pas vraiment être nazi. II est donc assez consternant de voir que les effets de cet acharnement apologétique ne s'estompent toujours pas. De nombreuses personnes qu'il est impossible de soupçonner de nazisme (tout comme Arendt elle-même) persistent à défendre un auteur aussi toxique que Heidegger au nom d'une transcendance supposée de la pensée sur l'Histoire, la politique et la société. Espérons que le livre exemplaire et salutaire de Emmanuel Faye éclaircira les esprits. Ces 
lumières sont d'autant plus nécessaires qu'il nous semble qu'Emmanuel Faye a entièrement raison d'écrire qu'Arendt " prépare et exprime à l'avance bien des ambivalences de la post-modernité » dans la mesure où elle " sait rendre acceptables les positions les plus contre-révolutionnaires et conservatrices en ajoutant quelques références - ici à Feuerbach, ailleurs à Rosa Luxemburg - qui les feront apparaître, à une lecture rapide, comme progressistes et révolutionnaires alors que, sur le fond, il n'en est rien » (Faye 2016 : 63). Il faudra tôt ou tard que les autres héritiers français de Heidegger, souvent indirects et se disant plutôt derridiens ou foucaldiens, se penchent de façon historique et critique sur l'origine et les implications de leurs thèmes directeurs. Emmanuel Faye leur en donne les moyens. Jusqu'ici rien n'autorise à être optimiste quant à leur capacité et à leur désir de s'en emparer.

\section{Bibliographie}

Adorno Theodor W. (2009). Jargon de l'authenticité. De l'idéologie allemande ( $1^{\text {re }}$ ed. 1964 ; traduit de l'allemand par Éliane Escoubas, 1989). Paris, Payot.

DOMERACKI Stéphane (2016). Heidegger et sa solution finale. Essai sur la violence de «la » «pensée ». Paris, Connaissances et Savoirs.

FAYE Emmanuel (2007). Heidegger, l'introduction du nazisme dans la philosophie (1 $1^{\text {è }}$ ed. Albin Michel 2005). Paris, Le Livre de Poche.

FAYE Emmanuel (2016). Arendt et Heidegger. Extermination nazie et déstruction de la pensée. Paris, Albin Michel.

Janicaud Dominique (2001). Heidegger en France, t. 1. Paris, AlbinMichel.

Kraus Karl (2005). Troisième nuit de Walpurgis. Marseille, Agone.

RASTIER François (2015). Naufrage d'un prophète. Heidegger aujourd'hui.

Paris, PUF. 